\title{
Slip avalanches in crystal plasticity: scaling of the avalanche cutoff
}

\author{
Michael Zaiser $\S$ and Nikos Nikitas \\ The University of Edinburgh, Institute for Materials and Processes, The King's \\ Buildings, Sanderson Building, Edinburgh EH9 3JL, UK
}

\begin{abstract}
Plastic deformation of crystals proceeds through a sequence of intermittent slip avalanches with scale-free (power-law) size distribution. On macroscopic scales, however, plastic flow is known to be smooth and homogeneous. In the present letter we use a recently proposed continuum model of slip avalanches to systematically investigate the nature of the cut-off which truncates scale-free behavior at large avalanche sizes. The dependence of the cut-off on system size, geometry, and driving mode, but also on intrinsic parameters such as the strain hardening rate is established. Implications for the observability of avalanche behavior in microscopic and macroscopic samples are discussed.
\end{abstract}

Keywords: plasticity, depinning, defects, avalanches.

$\S$ Tel. +44-131-6505671; Fax +44-131-6513470; e-mail M.Zaiser@ed.ac.uk 


\section{Introduction and Background}

It is now well established both experimentally (Weiss and Grasso 1997, Miguel et al. 2001, Dimiduk et. al. 2006, Richeton et al. 2006) and theoretically (Miguel et. al. 2001, Koslowski et. al. 2004, Zaiser and Moretti 2005) that plastic deformation of crystalline solids proceeds, on microscopic and mesoscopic scales, through an intermittent series of bursts ('slip avalanches') with scale-free size distribution. An overview of experimental and theoretical investigations of this phenomenon has been given recently by Zaiser (2006). Plastic strain increments $\Delta \epsilon$ produced by slip avalanches are power-law distributed, $p(\Delta \epsilon) \propto \Delta \epsilon^{-\kappa}$ where the exponent $\kappa$ is found to be approximately 1.5 (Miguel et. al. 2001, Zaiser and Moretti 2005, Dimiduk et. al. 2006). Acoustic emission experiments (Miguel et. al. 2001) indicate scale-free behavior over more than 8 orders of magnitude without any apparent cut-off.

This raises an intriguing question: If we consider a deformation curve (stress vs. strain in case of strain-controlled testing, stress vs. time in case of creep testing), then this curve will consist of a sequence of $N$ avalanches such that the total strain is given by $\epsilon_{\text {tot }}=N\langle\Delta \epsilon\rangle_{N}$ where $\langle\Delta \epsilon\rangle_{N}$ is the average strain increment produced by each of the $N$ events. We now evaluate the contribution $\Delta \epsilon_{\max }^{(N)} / \epsilon_{\text {tot }}$ of the largest event (of size $\Delta \epsilon_{\max }^{(N)}$ ) to the total deformation. For $p(\Delta \epsilon) \propto \Delta \epsilon^{-\kappa}$ with $1<\kappa<2$ we obtain the surprising result that in the limit $N \rightarrow \infty$ this ratio tends towards a finite value less than 1 . In other words, whatever the specimen size, the largest avalanche should always be directly visible on the macroscopic deformation curves. Experimentally, however, smooth behavior is observed on macroscopic scales and deformation bursts become apparent only when the specimen dimensions are reduced down to the micron scale.

Different explanations have been proposed to resolve this paradox. Zaiser and Moretti (2005) proposed the avalanche size to be limited by intrinsic hardening. Along similar lines, Richeton et. al. (2005) argued that the avalanche size in ice polycrystals may be limited by strong, grain-size dependent kinematic hardening induced by the strong plastic anisotropy of the material. Another possible explanation is that the avalanches might exhibit lamellar geometry (fractal dimension close to 2). Owing to the small volume involved in the deformation process, such avalanches would produce only a small macroscopic strain even if their magnitude is limited by the system size only. This idea is in agreement with traditional ideas about deformation localization in slip lines and slip bands (Neuhäuser 1983), but also with recent experimental observations which indicate that deformation localizes in lamellar regions with local strains distributed according to a power law with exponent close to 1.5 (Schwerdtfeger et. al. 2007). Unlike the conjecture of Zaiser and Moretti, this proposition predicts an extrinsic limit to the avalanche size, in line with the idea of self-organized criticality.

In the present letter we use the model of Zaiser and Moretti (2005) to clarify the respective influences of intrinsic (hardening) and extrinsic (specimen shape and size, driving mode) parameters on the size of deformation bursts in crystal plasticity. A short summary of the model is given in Section 2, together with its extension to include driving by an external 'machine' of variable stiffness. We then use the model to investigate avalanche size distributions and establish scaling relations which allow to estimate the size of the largest avalanches under various experimental conditions. 


\section{Description of the Model}

\subsection{Basic structure of the model}

We consider plastic deformation occurring by crystallographic slip on a single active slip system. Hence, the deformation state is completely characterized by the scalar shear strain field $\gamma(\boldsymbol{r})$. In the following we assume without loss of generality that the slip direction corresponds to the $x$ direction of a Cartesian coordinate system and the slip plane is the $x z$ plane. The driving force for plastic flow is the externally applied shear stress $\sigma_{x y}=: \tau_{\text {ext }}$ acting in this slip system. The evolution of the shear strain is governed by the equation

$$
\frac{1}{\chi} \partial_{t} \gamma=\tau_{\text {ext }}+\int \Gamma\left(\boldsymbol{r}-\boldsymbol{r}^{\prime}\right) \gamma\left(\boldsymbol{r}^{\prime}\right) \mathrm{d} \boldsymbol{r}^{\prime}+\delta \tau(\boldsymbol{r}, \gamma),
$$

which is formally equivalent to the equation of motion of a depinning elastic manifold. The fluctuating deformation resistance $\delta \tau(\boldsymbol{r}, \gamma)$ mimics the formation and dissolution of jammed dislocation configurations such as dipoles, multipoles, and (in 3D) dislocation junctions. It is understood as a random field with short-range correlations, $\left\langle\delta \tau(\boldsymbol{r}, \gamma) \delta \tau\left(\boldsymbol{r}^{\prime}, \gamma^{\prime}\right)=\left\langle\delta \tau^{2}\right\rangle f(\gamma-\right.$ $\left.\gamma^{\prime}\right) \delta\left(\boldsymbol{r}-\boldsymbol{r}^{\prime}\right)$ where $f$ is a rapidly decaying function. For details, see Zaiser and Moretti, 2005, and Zaiser and Aifantis, 2006.

The second term on the right-hand side of Eq. (1) describes long-range interactions mediated by the elasticity of the medium and characterized by the elastic Green's function $\Gamma$. In Fourier space, $\Gamma$ does not depend on the modulus of the wave vector, indicating an effectively infinite range of the interactions. An explicit calculation of this function has been given by Zaiser and Moretti (2005), but in the following we use an alternative formulation based on a dislocation representation of the stress field. For plane strain deformation $(\gamma=\gamma(x, y))$ with periodic boundary conditions we may write

$$
\frac{1}{\chi} \partial_{t} \gamma=\tau_{\text {ext }}-\int \tau\left(\boldsymbol{r}-\boldsymbol{r}^{\prime}\right) \partial_{x} \gamma\left(\boldsymbol{r}^{\prime}\right) \mathrm{d}^{2} r^{\prime}+\delta \tau(\boldsymbol{r}, \gamma),
$$

where $\tau(\boldsymbol{r})$ is the shear stress field of a straight edge dislocation of unit strength plus its periodic images. Similarly, for a general shear strain field $\gamma=\gamma(x, y, z)$ we may write

$$
\frac{1}{\chi} \partial_{t} \gamma=\tau_{\mathrm{ext}}-\int \tau_{\mathrm{e}}\left(\boldsymbol{r}-\boldsymbol{r}^{\prime}\right) \partial_{x} \gamma\left(\boldsymbol{r}^{\prime}\right) \mathrm{d}^{3} r^{\prime}-\int \tau_{\mathrm{s}}\left(\boldsymbol{r}-\boldsymbol{r}^{\prime}\right) \partial_{z} \gamma\left(\boldsymbol{r}^{\prime}\right) \mathrm{d}^{3} r^{\prime}+\delta \tau(\boldsymbol{r}, \gamma),
$$

where $\tau_{\mathrm{e}}$ and $\tau_{\mathrm{s}}$ are the shear stress fields generated by screw and edge dislocation segments of unit strength and unit length, plus their periodic images.

\subsection{Numerical Implementation}

We investigate the rate-independent limit $\chi \rightarrow \infty$ and implement an automaton version of the model on a square lattice, assuming plane strain geometry. The strain field takes discrete values $\gamma_{i j}$ at the lattice points. The long-range stress $\int \tau\left(\boldsymbol{r}-\boldsymbol{r}^{\prime}\right) \partial_{x} \gamma\left(\boldsymbol{r}^{\prime}\right) \mathrm{d} \boldsymbol{r}^{\prime}$ is evaluated as the sum of the stresses of edge dislocations of strength $\gamma_{i+1, j}-\gamma_{i j}$ that are placed midway between the lattice points in the $x$ direction. The stochastic fields $\delta \tau_{i j}=\delta \tau\left(\boldsymbol{r}_{i j}, \gamma_{i j}\right)$ are modelled as independent Ornstein-Uhlenbeck processes located at the respective lattice points. We allow positive strain increments only (rate-dependent implementations which allow the strain to locally decrease have been shown to yield similar results, see Zaiser 2006). In the simulations, the local strain at the lattice point $(i, j)$ is increased by a fixed amount $\Delta \gamma_{i j}=\Delta \gamma_{0}$ whenever the condition

$$
\tau_{\text {ext }}-\int \tau\left(\boldsymbol{r}_{i j}-\boldsymbol{r}^{\prime}\right) \partial_{x} \gamma\left(\boldsymbol{r}^{\prime}\right) \mathrm{d}^{2} r^{\prime}+\delta \tau_{i j}\left(\gamma_{i j}\right)>0
$$


is fulfilled. The strains are updated in parallel, and the new stresses are evaluated as detailed previously. The new deformation resistance is evaluated as

$$
\delta \tau_{i j}\left(\gamma_{i j}+\Delta \gamma_{i j}\right)=\alpha_{i j} \delta \tau\left(\gamma_{i j}\right)+\sqrt{1-\alpha_{i j}^{2}}\left\langle\delta \tau_{i j}^{2}\right\rangle w_{i j}
$$

where $\alpha_{i j}=\exp \left(-\Delta \gamma_{i j} / \gamma_{\text {corr }}\right), \gamma_{\text {corr }}$ is a correlation strain which characterizes the 'memory' of the Ornstein-Uhlenbeck process, $\left\langle\delta \tau_{i j}^{2}\right\rangle$ is the mean square amplitude of the pinning field, and the $w_{i j}$ are statistically independent Gaussian variables of unit variance. For the process defined by Eq. (5), the two-point correlation function is $\left\langle\delta \tau_{i j}\left(\gamma_{i j}\right) \delta \tau_{k l}\left(\gamma_{k l}^{\prime}\right)\right\rangle=$ $\left\langle\delta \tau_{i j}^{2}\right\rangle \delta_{i k} \delta_{j l} \exp \left[\left|\gamma_{i j}-\gamma_{k l}^{\prime}\right| / \gamma_{\mathrm{corr}}\right]$.

\subsection{External driving and strain hardening}

Strain hardening means that the resistance of a material to deformation increases with increasing strain. In our case, the deformation resistance is a random field with zero mean. Strain hardening can be implemented by increasing the amplitude of this field. To this end, we increase the local amplitude in Eq. (5) in proportion with the local strain, $\left\langle\delta \tau_{i j}^{2}\right\rangle=\left\langle\delta \tau_{0}^{2}\right\rangle\left(1+K \gamma_{i j}\right)^{2}$ where $K$ is a non-dimensional parameter that we use to adjust the average hardening rate.

In a strain-hardening system, sustained deformation requires a driving stress that on average increases with strain. In our simulations, we drive the system in a quasi-static manner, using either stress control or displacement control with a machine of finite stiffness. In a stresscontrolled simulation we simply increase the external stress $\tau_{\text {ext }}$ from zero in small increments $\Delta \tau_{\text {ext }}$. After each stress increment we check for all volume elements whether Eq. (4) is fulfilled. At the 'unstable' sites where this is the case, we increase the local strains by $\Delta \gamma_{0}$ and re-evaluate the local deformation resistances. After all sites are updated, we re-evaluate the local stresses everywhere in the system and perform another update. This is repeated until all sites are stable (Eq. (4) is no longer fulfilled for any site). Then we again increase the external stress, and so on.

In a displacement-controlled test, we impose the total deformation $\gamma_{\text {ext }}$ and evaluate the stress according to

$$
\tau_{\mathrm{ext}}=M\left[\gamma_{\mathrm{ext}}-\left\langle\gamma_{i j}\right\rangle\right],
$$

where $M$ is called the 'machine stiffness'. In a quasi-static simulation we increase $\gamma_{\text {ext }}$ (and thus the stress) at a small rate until at least one site fulfils Eq. (4) and becomes unstable. We then keep $\gamma_{\text {ext }}$ fixed while we update in parallel the strains at all unstable sites as previously outlined. However, after each update we not only re-evaluate the local stresses and deformation resistances, but also the external stress which, owing to the increase of $\left\langle\gamma_{i j}\right\rangle$ during the update, is bound to decrease according to Eq. (6). Once all sites have stabilized, we again increase the imposed strain, and so on. The procedure can be visualized as 'pulling' the system with a spring of finite stiffness: As soon as the system yields, the spring is partly relaxed and the driving force decreases by an amount that is proportional to the spring stiffness.

\subsection{Relation with physical parameters of dislocation systems}

The model as stated above applies to the shear deformation of any disordered material. When the disorder relates to the dislocation arrangement in a deforming crystal, scaling relations can be used to relate the model parameters to physical variables of the system. To this end, we consider a dislocation system of density $\rho$ and note that the characteristic correlation 
length of internal stress fluctuations in such systems is of the order of one dislocation spacing $\rho^{-1 / 2}$ (for a detailed discussion, see Zaiser and Aifantis 2006). The volume occupied by one lattice site can be identified with the characteristic volume occupied by a dislocation segment, $V_{0}=\rho^{-3 / 2}$. The correlation strain $\gamma_{\text {corr }}$ corresponds to the strain accomplished locally when a dislocation segment crosses this elementary volume, $\gamma_{\text {corr }}=b \sqrt{\rho}$ where $b$ is the modulus of the Burgers vector of the dislocations. This also defines the natural unit of strain in a dislocation system. The deformation resistance $\delta \tau$, as all other stresses, scales like $G b \sqrt{\rho}$ where $G$ is the shear modulus. From the scaling of stress and strain it follows that the 'natural unit' of the hardening rate and the machine stiffness is simply the shear modulus $G$.

In the following all quantities will be given in these 'natural units'. The size $s$ of a strain burst is defined as the sum of all strain increments that occur during a period of activity. The corresponding macroscopic strain is given by $s / X Y$ where the integers $X$ and $Y$ define the size of the lattice in the $x$ and $y$ directions.

\section{Results}

As discussed by Zaiser and Moretti (2005), in the absence of hardening and under conditions of stress control, the model shows a 'phase transition' between a pinned and a moving phase. This transition occurs at a critical stress level which defines the yield stress of the system. In the following we choose the initial value of $\left\langle\delta \tau_{0}^{2}\right\rangle$ such that this yield stress is about 0.3 (dimensional value $0.3 G b \sqrt{\rho}$ ), in line with typical experimental findings in fcc metals. The correlation strain is set equal to unity, and the parameter $K$ is used to tune the hardening rate $H$. We also systematically vary the machine stiffness $M$ and the system size. There are no other parameters in the model.

Figure 1 shows three deformation curves (external stress vs. average strain) obtained for different values of the machine stiffness and hardening rate. In a stress-controlled simulation

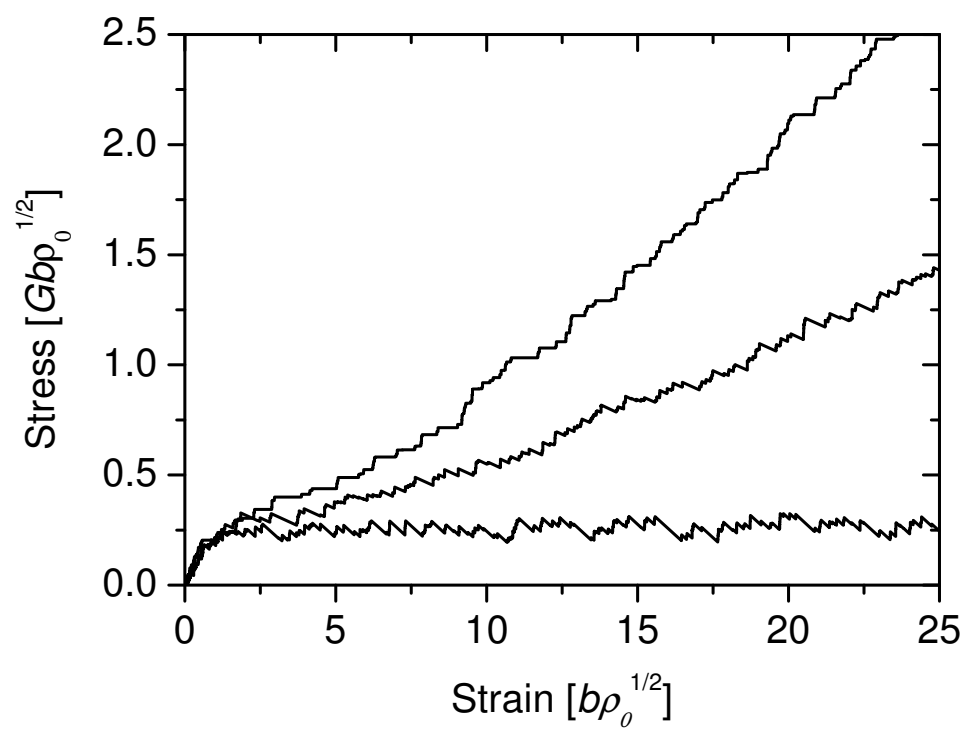

Figure 1. Stress-strain curves as obtained from simulation of a system with $64 \times$ 64 sites; from top to bottom: hardening rate $H=0.125$, machine stiffness $M=0$; hardening rate $H=0.0625$, machine stiffness $M=0.0625$, hardening rate $H=0$, machine stiffness $M=0.125$. 
and in the absence of hardening, the external stress passes through an initial transient until it reaches the yield stress, at which point the strain increases indefinitely. In a displacementcontrolled simulation, on the other hand, the external stress fluctuates slightly below the yield-stress level: Close to the yield stress, slip avalanches are triggered which decrease the external stress, after an avalanche is terminated the external stress rises again, and so on. In the presence of hardening, the mean stress after the initial transient increases first slowly ('Stage I') and then at a higher, approximately constant rate ('Stage II'). Statistics of slip avalanches were determined in this constant-hardening stage (above a strain of approximately 10 in Figure 1). The hardening rate $H$ was defined as the average slope of the stress-strain curve in this regime, with the average either determined from the stress-strain curve of a very large system or from the average of many smaller systems. Both methods were found to yield similar results.

\begin{tabular}{|l|l|l|l|}
\hline series & fixed parameters & varied parameter & variation range \\
\hline 1 & $M=1, H=0$ & system size & $32 \times 32 \ldots 128 \times 128$ \\
\hline 2 & $M=0.5, H=0$ & system size & $16 \times 16 \ldots 128 \times 128$ \\
\hline 3 & $M=0.25, H=0$ & system size & $32 \times 32 \ldots 128 \times 128$ \\
\hline 4 & $M=0.125, H=0$ & system size & $64 \times 64 \ldots 128 \times 128$ \\
\hline 5 & $M=0, H=0.0065$ & system size & $32 \times 32 \ldots 128 \times 128$ \\
\hline 6 & $M=0, H=0.013$ & system size & $32 \times 32 \ldots 128 \times 128$ \\
\hline 7 & $M=0, H=0.026$ & system size & $32 \times 32 \ldots 256 \times 256$ \\
\hline 8 & $M+H=0.125, X=Y=64$ & stiffness $M$ & $0 \ldots 0.125$ \\
\hline 9 & $M=0.125, H=0, X=16$ & extension $Y$ & $16 \ldots 128$ \\
\hline 10 & $M=0.125, H=0, Y=16$ & extension $X$ & $16 \ldots 128$ \\
\hline
\end{tabular}

Table 1. Series of simulations carried out in this study. Scans over the variation ranges were performed by changing the respective parameters by a factor of two between two simulations in a series.

\subsection{Truncation of avalanches due to machine-induced stress relaxation}

To determine avalanche statistics, several series of simulations were carried out (Table 1). Generally, the avalanche size distributions possess the scaling form

$$
p(s) \propto s^{-3 / 2} f\left(s / s_{0}\right)
$$

where $s_{0}$ is the characteristic avalanche size and the function $f$ can be well approximated by $f\left(s / s_{0}\right) \approx \exp \left[-\left(s / s_{0}\right)^{2}\right]$. Distributions corresponding to series 1-4 on Table 1 are shown in Figure 2. It can be seen that an increase in machine stiffness $M$ leads to a decrease and an increase of system size to an increase in $s_{0}$. This is to be expected, since the machine decreases the driving stress, during an avalanche of size $s$, by an amount $M s / N$ where $N$ is the number of lattice sites. This causes large avalanches to self-terminate. A more quantitative analysis can be performed by fitting Eq. (7) to the distributions to determine the characteristic size $s_{0}$ as a function of the simulation parameters. Interestingly, it turns out that $s_{0}$ scales as $s_{0} \propto L / M$ where $L$ is the linear dimension of the system, rather than $s_{0} \propto N / M \propto L^{D} / M$ where $D$ is the system dimension. 


\subsection{Equivalence of hardening and machine-induced stress relaxation}

Hardening locally increases the pinning strength of the system at those sites that are actually involved in a slip avalanche. Machine-induced stress relaxation decreases the global driving

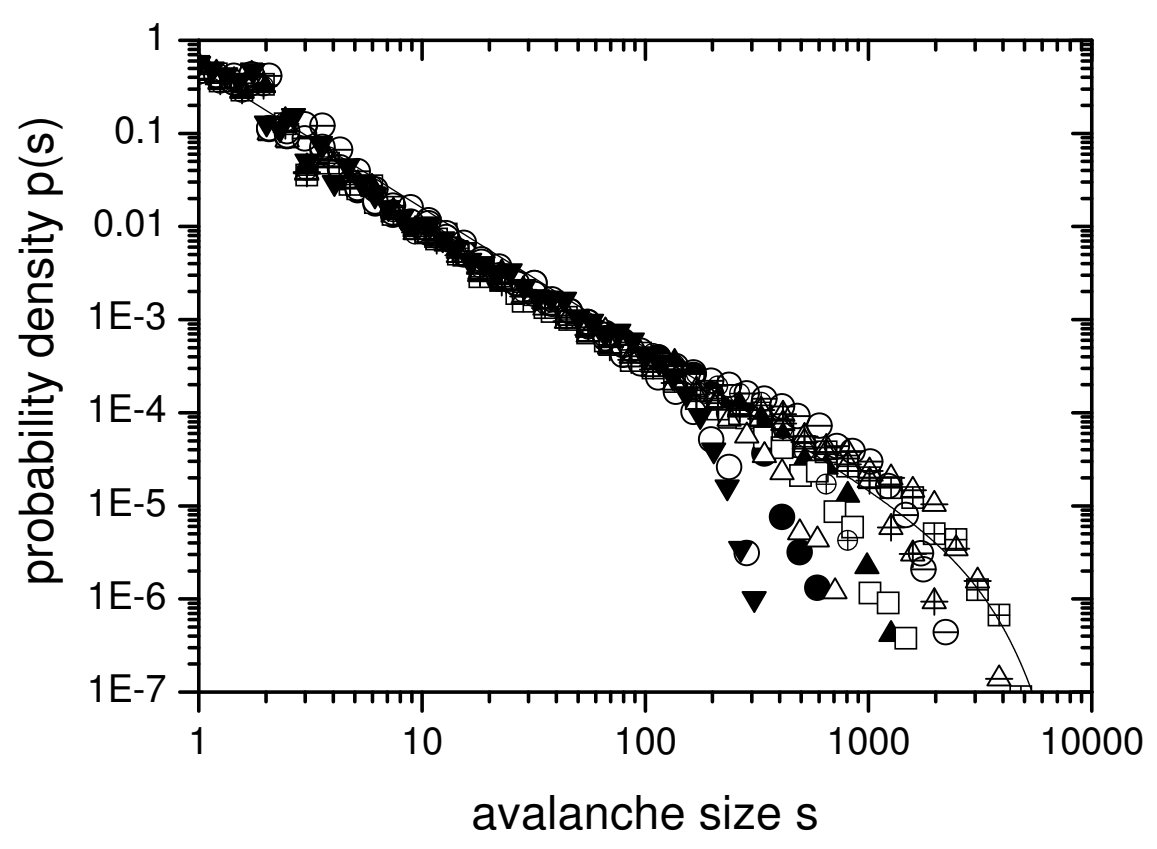

Figure 2. Avalanche size distributions corresponding to simulation series 1 (full symbols), series 2 (open symbols), series 3 (cross-centered symbols) and series 4 (barcentered symbols). Symbol shape indicates system size: square $128 \times 128$, up triangle $64 \times 64$, circle $32 \times 32$, down triangle $16 \times 16$.

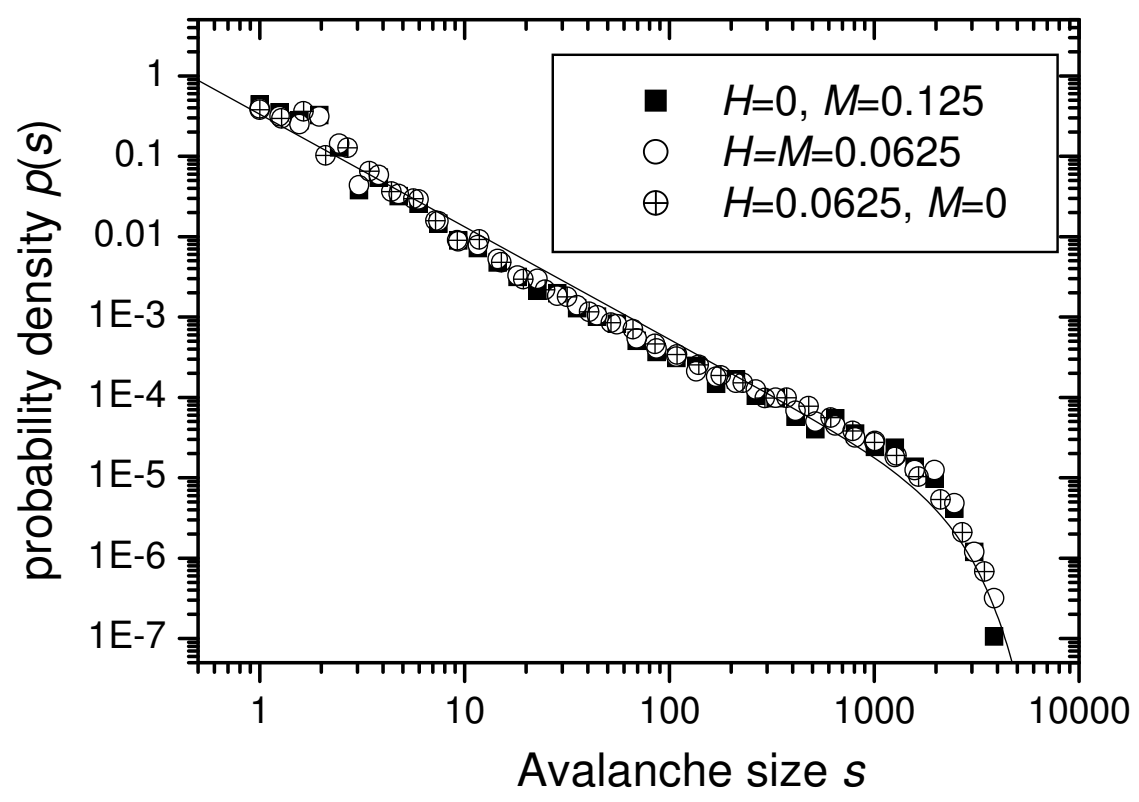

Figure 3. Avalanche size distributions for three different systems of size $64 \times 64$; Parameters $M$ and $H$ see inset; Full line: fit according to Eq. (7), with $s_{0}=1700$. 
force on all sites. Both effects should have equivalent consequences for the avalanche statistics if the depinning transition of the system shows mean-field behavior.

Figure 3 shows avalanche size distributions determined from an ensemble of simulations similar to those shown in Figure 1. Different machine stiffnesses and hardening rates were imposed such that the sum $M+H$ was the same for all simulations. It can be seen that three sets of simulations ( $M=0, H=0.125 ; M=H=0.0625 ; M=0.125, H=0)$ yield exactly the same distribution of avalanche sizes, which is well described by the scaling form (7). This demonstrates that strain hardening and machine-induced stress relaxation indeed have similar effects on the avalanche dynamics. As a consequence, the avalanche cutoff $s_{0}$ depends on the sum $H+M$ of the hardening rate and machine stiffness, rather than on $H$ and $M$ separately.

\subsection{Scaling law for the characteristic avalanche size}

After performing several series of simulations at different hardening rates (series 5-7 in Table 1) and determining the characteristic size $s_{0}$ for each set of parameters, it was found that the scaling of the cut-off can, over the entire parameter range investigated, be described by the general scaling law

$$
s_{0}=\frac{C L}{(M+H)} .
$$

A fit to all data yields $C \approx 5$ (Figure 4 ). We also performed simulations for anisotropic specimen shapes. In this case we found approximately the same scaling law, with $L$ defined as the square root of the number of lattice sites (Series 9 and 10 in Figure 4).

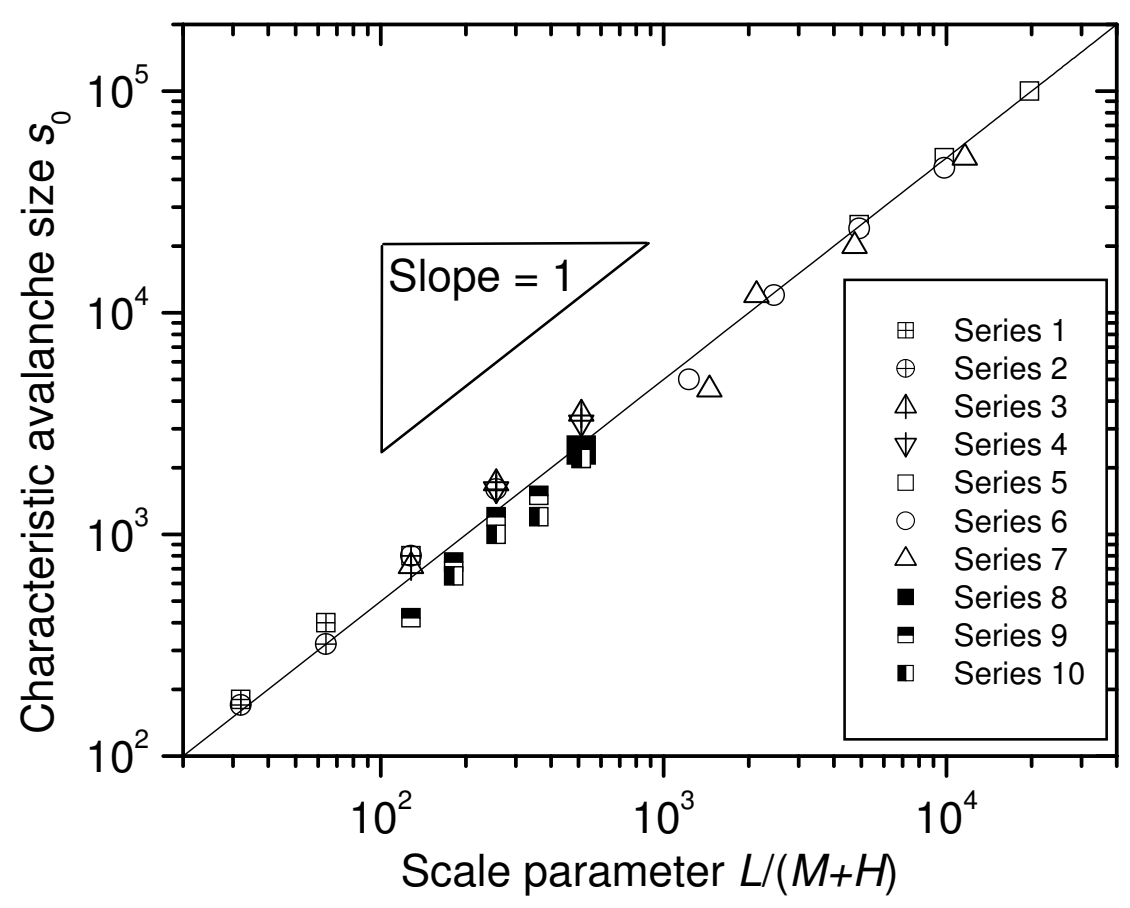

Figure 4. Scaling of the avalanche cutoff with hardening rate $H$, machine stiffness $M$, and linear system dimension $L$. Full line: $s_{0}=5 L /(M+H)$. 


\section{Discussion and Conclusions}

Our results have been obtained in the limit of quasi-static driving, i.e., the applied stress or the imposed displacement are kept fixed during an avalanche. This is the relevant limit for dislocation systems. Direct observation of deformation by surface monitoring indicates an extremely high level of intermittency in dislocation plasticity, in the sense that a given small volume element is plastically inactive almost all of the time: the instantaneously active slip volume is of the order of $10^{-7}$ of the macroscopic crystal volume, see Neuhäuser (1984) and Zaiser (2000).

To assess the implications of our results for such 'real' systems, it is useful to revert to dimensional coordinates. The average strain increment produced by an avalanche of size $s_{0}$ is given by $s_{0} / N$. In dimensional units, this becomes $\Delta \gamma_{\max }=C b G / l(H+M)$ where $l$ is the (dimensional) specimen length. It is even more instructive to evaluate the length increment $\Delta l_{\text {max }}$ produced by such an avalanche during axial loading. Using that the axial plastic strain $\epsilon$ is related to the shear strain by $\epsilon=\Delta l / l=m \gamma$ where $m$ is the Schmid factor, we find that

$$
\Delta l_{\max }=\frac{C b G m}{(M+H)} .
$$

This compares well with experimental data on micron-sized single crystals. Dimiduk et. al. (2006) determined the statistics of elongation bursts observed during compression of $\mathrm{Ni}$ microcrystals. Specimens with sizes of the order of 20 micrometers were tested under conditions of load control (the load was kept constant during a burst). Typical hardening rates found in these experiments were of the order of $\mathrm{d} \tau_{\text {ext }} / \mathrm{d}\langle\gamma\rangle \approx G / 1500$. With $C=5, b=2.5 \times 10^{-10}$ $\mathrm{m}$, and $m \approx 0.4$, we find that the length increments caused by the largest bursts are expected to be about 0.7 microns. This compares very well with the maximum burst sizes observed by Dimiduk et. al. More importantly, the simple scaling relation predicted in the present work can be easily verified by comparing specimens of different sizes (the largest elongation bursts should be roughly independent on specimen size), and by comparing results obtained in stress and displacement control.

The present results offer a simple explanation of the fact that avalanches are not observed in macroscopic deformation. Length increments of the order of less than a micron cannot be resolved by standard macroscopic testing equipment. In macroscopic samples with sizes of the order of $10^{-1} \mathrm{~m}$, such length increments correspond to axial strain increments $\Delta \epsilon$ of less than $10^{-5}$. The proposed scaling relation is consistent with the idea of a lamellar, system-spanning 'shape' of the largest avalanches: The prediction that the average strain produced by these avalanches decreases in proportion with the linear dimension of the specimen follows naturally from the assumption that each avalanche corresponds to the formation of a lamellar slip line or slip band. However, effects of machine stiffness and/or intrinsic hardening are relevant in delimiting the avalanche size by controlling the amount of strain produced within such an active region.

\section{Acknowledgements}

Financial support of the European Commission under NEST-2005-PATH-COM-043386 and of EPSRC under Grant No. EP/E029825 is gratefully acknowledged. 


\section{References}

Dimiduk, D.M., Woodward, C., LeSar, R., Uchic, M.D., 2006. Scale-Free Intermittent Flow in Crystal Plasticity, Science 26, 1188-1190.

Koslowski, M., LeSar, R., Thomson, R., 2004. Avalanches and scaling in plastic deformation, Phys. Rev. Letters 93, 125502.

Miguel, M.C., Vespignani, A., Zapperi, S., Weiss, J., Grasso, J.-R., 2001. Intermittent dislocation flow in viscoplastic deformation. Nature 410, 667-671.

Neuhäuser, H., 1984. Slip-line formation and collective dislocation motion. In: F.R.N. Nabarro (Ed.), Dislocations in Solids, Vol. 4, North-Holland, Amsterdam, pp. 319-440.

Richeton, T., Weiss, J., and Louchet, F., 2005. Breakdown of avalanche critical behaviour in polycrystalline plasticity. Nature Materials 4, 465-469.

Richeton, T., Dobron, P., Chmelik, F., Weiss, J., and Louchet, F., 2006. On the critical character of plasticity in metallic single crystals. Mater. Sci. Engng. A 424, 190-195.

Schwerdtfeger, J., Nadgorny, E.M., Madani-Grasset, F., Koutsos, V., Blackford, J.R., Zaiser, M., 2007. Scale-free statistics of plasticity-induced surface steps on $\mathrm{KCl}$ single crystals. J. Stat. Mech., submitted.

Weiss, J., Grasso, J.-R., 1997. Acoustic emission in Single Crystals of Ice. J. Phys. Chem. B 101, 6113-6117.

Zaiser, M., Moretti, P., 2005. Fluctuation phenomena in crystal plasticity - a continuum model. J. Stat. Mech, P08004.

Zaiser, M., Aifantis, E.C., 2006. Randomness and slip avalanches in gradient plasticity. Int. J. Plasticity 22, 1432-1455.

Zaiser, M., 2000. Statistical modelling of dislocation systems, Materials Science and Engineering A 309/310, 304-315

Zaiser, M., 2006. Scale invariance in plastic flow of crystalline solids. Adv. Physics 55, $185-245$. 